\title{
Measurable process selection theorem and non-autonomous inclusions
}

\author{
Jorge E. Cardona and Lev Kapitanski \\ Department of Mathematics, University of Miami, Coral Gables, FL 33124, USA
}

\begin{abstract}
A semi-process is an analog of the semi-flow for non-autonomous differential equations or inclusions. We prove an abstract result on the existence of measurable semiprocesses in the situations where there is no uniqueness. Also, we allow solutions to blow up in finite time and then obtain local semi-processes.
\end{abstract}

\section{Introduction}

Let

$$
\frac{d u}{d t}=f(u)
$$

be an archetypical autonomous differential equation. Autonomous refers to the structure of the equation and means that the independent variable, $t$, does not appear explicitly (independently) in the equation. Because of that, (1) is invariant under the time shift (translation) $\theta_{\tau}: t \mapsto t+\tau$, and if $u(\cdot)$ is a solution of (1), then $\theta_{\tau} u(\cdot)=u(\cdot+\tau)$ is a solution as well. Suppose (1) describes evolution/dynamics on some set $X$ (which could be a finiteor infinite-dimensional vector space or manifold). Given an $a \in X$, let $u(t, a), t \in[0,+\infty)$ be a solution of (11) starting at $u(0, a)=a$ (let us assume that global solutions exist forward in time). If $v\left(\cdot, u\left(t_{1}, a\right)\right)$ is a solution of (11) starting (at $\left.t=0\right)$ from the point $u\left(t_{1}, a\right)$, we can splice $u$ and $v$ and obtain a (possibly new) solution $w=u \underset{t_{1}}{\bowtie}$ starting at $a$ :

$$
w(t)=u \bowtie v(t)= \begin{cases}u(t, a), & \text { if } 0 \leq t \leq t_{1}, \\ v\left(t-t_{1}, u\left(t_{1}, a\right)\right), & \text { if } t \geq t_{1} .\end{cases}
$$

If solutions are unique (for every $a \in X$ there is a unique solution $u(t, a)$ ), then $v\left(t, u\left(t_{1}, a\right)\right)=$ $u\left(t+t_{1}, a\right)$. In general, in the case of uniqueness, the solutions of (1) enjoy the semigroup property, i.e., for every $a \in X, u(0, a)=a$ and

$$
u\left(t_{2}, u\left(t_{1}, a\right)\right)=u\left(t_{1}+t_{2}, a\right), \quad \forall t_{1}, t_{2} \geq 0 .
$$


This allows us to define the semigroup $U(t): X \rightarrow X$ by the formula $U(t)(a)=u(t, a)$.

For non-autonomous differential equations the situation is similar and different. Consider an archetypical non-autonomous equation

$$
\frac{d u}{d t}=g(t, u)
$$

Now, in addition to the initial position/state $a \in X$, it is important to specify the initial moment of time, $t_{0}$. The solution(s) will depend on $a$ and $t_{0}$; we write $u\left(t ; t_{0}, a\right)$ to denote a solution of (44) for $t \geq t_{0}$ that equals $a$ when $t=t_{0}$. If we follow the solution $u\left(t ; t_{0}, a\right)$ until the moment $t=t_{1}$ and then follow a solution $v\left(t ; t_{1}, u\left(t_{1} ; t_{0}, a\right)\right)$ that starts at the point $u\left(t_{1} ; t_{0}, a\right)$ at the moment $t_{1}$, we obtain a spliced solution of (44),

$$
w\left(t ; t_{0}, a\right)=u \bowtie v(t)= \begin{cases}u\left(t ; t_{0}, a\right), & \text { if } t_{0} \leq t \leq t_{1}, \\ v\left(t ; t_{1}, u\left(t_{1} ;, t_{0}, a\right)\right), & \text { if } t \geq t_{1} .\end{cases}
$$

If the solutions of equation (44) are unique, we have the following analog of the semigroup property:

$$
u\left(t_{0} ; t_{0}, a\right)=a \quad \text { and } \quad u\left(t_{2} ; t_{1}, u\left(t_{1} ; t_{0}, a\right)\right)=u\left(t_{2} ; t_{0}, a\right), \quad \forall a \in X \forall t_{2} \geq t_{1} \geq t_{0} \geq 0 .
$$

Also, we can define the transition map $U\left(t_{1} ; t_{0}\right)$ that maps $X$ into $X$ by assigning to every $a \in X$ the value $u\left(t_{1} ; t_{0}, a\right)$ of the solution $u\left(t ; t_{0}, a\right)$. This transition map has the properties

$$
\begin{aligned}
& U(t ; t)=\operatorname{id}_{X}, \forall t \geq 0 \\
& U\left(t_{2} ; t_{1}\right) \circ U\left(t_{1} ; t_{0}\right)=U\left(t_{2} ; t_{0}\right), \forall t_{2} \geq t_{1} \geq t_{0} \geq 0 .
\end{aligned}
$$

A family of maps $U\left(t_{1} ; t_{0}\right)$ with the properties (7) will be called a process (see, e.g., [7, 12]). To the autonomous case correspond homogeneous processes characterized by time invariance: $U\left(t_{1}-\tau ; t_{0}-\tau\right)=U\left(t_{1} ; t_{0}\right)$ for all (admissible) $\tau$ (and therefore $U(t)=U(t ; 0)$ is the semigroup).

It may be advantageous to think of solutions of the autonomous equation (11) as integral curves (trajectories) in $X$, i.e., view solitons as continuous (infinite, one-sided) paths in $X$. If there is no uniqueness, the solutions/integral curves starting at the point $a$ form an integral funnel, $S(a)$, a subset of all paths starting at $a$. (Analysis of integral funnels for ODEs was initiated by H. Kneser in the 1920s, [16].) Denote by $\Omega$ the space of all continuous (infinite, one-sided) paths in $X$. Then the map $a \mapsto S(a)$ is a set-valued map (other names: multifunction, correspondence) from $X$ into $2^{\Omega}$. It has the (already mentioned) properties: if the path $w$ is in $S(a)$, then its shift, $\theta_{\tau} w$ is in $S(w(\tau))$, and if $u \in S(a)$ and $v \in S\left(u\left(t_{1}\right)\right)$, then $u \bowtie t_{t_{1}} v \in S(a)$.

An interesting and important question is whether it is possible to select a solution $u(\cdot, a)$ from every funnel $S(a)$ in such a way, that $u(t, a)$ has the semigroup property (3). In other words, is it possible to define a semigroup (semiflow) $U(t)$ so that, for every $a \in X$, 
$u(t, a)=U(t)(a), t \geq 0$, is a path in $S(a)$ ? In [5, 6], we show that the answer is yes under some very general assumptions. Moreover, we show that the selection $a \mapsto u(\cdot, a) \in S(a)$ is measurable. This is a new type of selection theorems (for measurable selection results see, e.g., [1, Section 18.3], [13], and the surveys [25, 14]). Our results were motivated by the Markov selection theorems, see [17, 24, 8, 9].

Here, we extend the semiflow selection theorem of [5, 6] to non-autonomous equations and processes. If there is no uniqueness for (4), but the solutions to the initial-value problem exist forward in time, we have the integral funnels $S\left(t_{0}, a\right)$ formed by all the solution $u\left(t ; t_{0}, a\right)$, $t \geq t_{0}$, such that $u\left(t_{0} ; t_{0}, a\right)=a$. The question we ask is whether there exists a measurable selection $\left(t_{0}, a\right) \mapsto u\left(\cdot ; t_{0}, a\right) \in S\left(t_{0}, a\right)$ such that $u\left(t ; t_{0}, a\right)$ satisfies (6) $)$. The answer again is yes under the right assumptions. After our previous work [5, 6], this answer is not surprising if we are concerned with solutions of the non-autonomous equation (4): one could replace (4) with the equivalent autonomous system

$$
\frac{d u}{d s}=g(t, u), \quad \frac{d t}{d s}=1
$$

Then, if we apply our results from [5, 6] and obtain a semigroup $\tilde{U}(s), s \geq 0$, on $X \times[0,+\infty)$ corresponding to (8) , the maps $U\left(t_{1}, t_{0}\right)(a)=\tilde{U}\left(t_{1}\right)\left(a, t_{0}\right)$ would form a process corresponding to (4). However, if we are in a more general setting and deal with integral funnels, their autonomisation is not clear. Thus, in the next section we present the precise statement and the proof of the existence of a measurable process. After that, in Section 3 we study the existence of local processes. These apply to the situations, where in addition to nonuniqueness we allow solutions to blow up in finite time. [In the 1960-70s there was some interest in abstracting the dynamical and semi-dynamical systems and processes, see, e.g., [10, 11, 3]. Our approach involves funnels and is different.] An example on the semi-process selection is presented in Section 4 .

A different and important point of view on non-autonomous dynamics involves the skewproduct construction, see, e.g., [21, 22]. The skew-product set-up is very convenient for the study of the long term behavior of non-autonomous systems. However, for the measurable processes selection, we believe our direct approach is more natural. We should mention that our abstract results apply not only to non-autonomous ordinary differential equations, but to partial differential equations, to differential and difference inclusions, and to other situations where integral funnels make sense.

\section{Global processes}

Let $X$ be a separable complete metric space with metric $\rho$, which we assume to be bounded: $\rho(x, y) \leq 1$ for all $x, y \in X$. Denote by $\mathcal{B}_{X}$ the Borel $\sigma$-algebra of $X$. Let $\Omega$ be the space of all continuous infinite one-side paths in $X$ equipped with the compact-open topology. The elements of $\Omega$ are continuous maps $u:[0,+\infty) \rightarrow X$, and convergence in $\Omega$ is the uniform convergence on every finite time interval. The space $\Omega$ is Polish; we fix a complete bounded 
metric on $\Omega$ by setting

$$
d(u, v)=\sum_{\ell=1}^{\infty} 2^{-\ell} \sup _{t \in[0, \ell]} \rho(u(t), v(t))\left[1+\sup _{t \in[0, \ell]} \rho(u(t), v(t))\right]^{-1} .
$$

Sometimes it is convenient to view the paths parametrized by $s \in[\tau,+\infty)$. We denote by $\Omega^{\tau}$ the space of continuous maps from $[\tau,+\infty)$ into $X$. It can be identified with the image of $\Omega$ under the (past erasing) map $\sigma_{\tau}$ : if $u(t), t \geq 0$, is a path in $\Omega$, then the path $\sigma_{\tau} u:[\tau,+\infty) \rightarrow X$ is defined as $\sigma_{\tau} u(t)=u(t)$ for $t \geq \tau$. On the other hand, $\Omega^{\tau}$ can be viewed as a subset of $\Omega$ if we extend the paths $v(t)$ in $\Omega^{\tau}$ to $[0, \tau)$ as staying at $v(\tau)$ :

$$
\partial_{\tau}: \Omega^{\tau} \rightarrow \Omega, \quad\left(\partial_{\tau} v\right)(t)= \begin{cases}v(\tau) & \text { when } 0 \leq t<\tau, \\ v(t) & \text { when } t \geq \tau .\end{cases}
$$

We will also use the notation $\Omega_{a}^{\tau}$ for all the paths in $\Omega^{\tau}$ staring at the point $a$, i.e., $v(\tau)=a$ if $v \in \Omega_{a}^{\tau}$. On occasion, it will be convenient to specify $\tau$ and $a$ in the notation for a path, e.g., $v(t ; \tau, a)$.

The integral funnels $S\left(t_{0}, a\right)$ will be subsets of the set $\Omega_{a}^{t_{0}}$. Denote by $P_{c l}[\Omega]$ the space of all (bounded) closed subsets of $\Omega$ endowed with the Vietoris topology (= exponential topology), see [18], [2], and 11] for details on set-valued maps, their properties and, in particular, measurability. In this presentation, all set-valued maps from $X$ to $\Omega$ will have non-empty closed values and will be viewed as maps from the measurable space $\left(X, \mathcal{B}_{X}\right)$ to $P_{c l}[\Omega]$ with the Vietoris topology. If $\Gamma$ is such a map and $A \subset \Omega$, define

$$
\Gamma^{-}(A)=\{x \in X: \Gamma(x) \cap A \neq \emptyset\} .
$$

For historical reasons, there are several confusingly similar notions of measurability of setvalued maps. A map $\Gamma: X \rightarrow P_{c l}[\Omega]$ is weakly measurable if $\Gamma^{-}(G) \in \mathcal{B}_{X}$ for every open set $G \subset \Omega . \Gamma$ is measurable if $\Gamma^{-}(F) \in \mathcal{B}_{X}$ for every closed set $F \subset \Omega$. Since $\Omega$ is metric, if $\Gamma$ is measurable, it is weakly measurable, [1, Lemma 18.2]. Since $\Omega$ is in addition separable, if $\Gamma$ is compact-valued and weakly measurable, it is measurable, [1, Theorem 18.10]. Thus, in our setting, for compact-valued $\Gamma: X \rightarrow P_{c l}[\Omega]$, there is no difference between weak measurability and measurability.

The fundamental result of Kuratowski and Ryll-Nardzewski, [19, Theorem 1, p. 398], implies that if $\Gamma: X \rightarrow P_{c l}[\Omega]$ is weakly measurable, then $\Gamma$ has a measurable selection, i.e., there exists a single-valued map $\gamma: X \rightarrow \Omega$ such that $\gamma(x) \in \Gamma(x)$ for all $x \in X$ and $\gamma$ is $\left(\mathcal{B}_{X}, \mathcal{B}_{\Omega}\right)$-measurable: for every Borel set $A \subset \Omega, \gamma^{-1}(A)$ is a Borel set in $X$.

We introduce now abstract integral funnels $S\left(t_{0}, a\right)$ that have the properties prompted by the properties of integral funnels of solutions of equation (4).

Definition 1. $S\left(t_{0}, a\right)$, where $t_{0} \in[0,+\infty)$ and $a \in X$, is a family of abstract integral funnels on the space $X$ if, for every $t_{0} \geq 0, S\left(t_{0}, \cdot\right): X \rightarrow P_{c l}[\Omega]$ is a set-valued map with the following properties. 
S1 For every $a \in X, S\left(t_{0}, a\right)$ is a non-empty compact subset of $\Omega_{a}^{t_{0}}$. Every path $u$ in $S\left(t_{0}, a\right)$ is parametrized as $u\left(t ; t_{0}, a\right)$, where $t \geq t_{0}$.

S2 Each map $S\left(t_{0}, \cdot\right)$ is measurable, i.e., for every closed set $C \subset \Omega^{t_{0}}$,

$$
\left\{x \in X: S\left(t_{0}, x\right) \cap C \neq \emptyset\right\} \in \mathcal{B}_{X}
$$

S3 If $u \in S\left(t_{0}, a\right)$, then $\sigma_{\tau} u \in S\left(t_{0}+\tau, u\left(t_{0}+\tau\right)\right)$.

S4 If $u \in S\left(t_{0}, a\right)$ and $v \in S\left(t_{0}+\tau, u\left(t_{0}+\tau\right)\right)$, then the spliced path $w=u \underset{t_{0}+\tau}{\bowtie} v$, defined as in (5), belongs to the funnel $S\left(t_{0}, a\right)$.

Theorem 1. Every family of abstract integral funnels $S\left(t_{0}, a\right), t_{0} \geq 0, a \in X$, has, for every $t_{0}$, a measurable selection $a \rightarrow u\left(\cdot ; t_{0}, a\right) \in S\left(t_{0}, a\right)$ with the semigroup property (6). As a corollary, there is a Borel measurable (semi)process $U\left(t_{1}, t_{0}\right): X \rightarrow X$ whose orbits are $U\left(t_{1}, t_{0}\right)(a)=u\left(t_{l} ; t_{0}, a\right)$, for all $t_{1} \geq t_{0} \geq 0$ and for all $a \in X$.

Proof. We modify our proof for the autonomous case from [6]. The plan is to successively reduct each funnel $S\left(t_{0}, a\right)$ while preserving the properties $\mathbf{S} \mathbf{1}-\mathbf{S} \mathbf{4}$ and so that the limiting funnel would contain just one path; property S3 for the limiting funnel then spells (6 6 ). The reduction of the funnels is based on an idea from optimization theory that was used by $\mathrm{N}$. V. Krylov in his proof of the Markov selection theorem, [17].

Let $\varphi: X \rightarrow[0,1]$ be a continuous function and let $\lambda$ be a positive real number. For $t_{0} \geq 0$ and $a \in X$, define the functional $\zeta$ on $S\left(t_{0}, a\right)$ via the formula

$$
\zeta(w)=\int_{0}^{\infty} e^{-\lambda t} \varphi\left(w\left(t_{0}+t\right)\right) d t
$$

This is a continuous functional and it attains its maximum on the compact set $S\left(t_{0}, a\right)$. Denote this maximum by $m_{\zeta}\left(t_{0}, a\right)$,

$$
m_{\zeta}\left(t_{0}, a\right)=\max _{w \in S\left(t_{0}, a\right)} \zeta(w)
$$

[The function $m_{\zeta}\left(t_{0}, \cdot\right): X \rightarrow \mathbb{R}$ is called the value function.] Define

$$
V_{\zeta}\left[S\left(t_{0}, a\right)\right]=\left\{v \in S\left(t_{0}, a\right): \zeta(v)=m_{\zeta}\left(t_{0}, a\right)\right\} .
$$

By the so-called measurable maximum theorem, [1, Theorem 18.19], $V_{\zeta}\left[S\left(t_{0}, a\right)\right]$ is a nonempty compact subset of $S\left(t_{0}, a\right)$, and the set-valued map $a \mapsto V_{\zeta}\left[S\left(t_{0}, a\right)\right]$ is measurable. Thus, the family of sets $V_{\zeta}\left[S\left(t_{0}, a\right)\right]$ has properties $\mathbf{S} 1$ and $\mathbf{S} 2$ of the abstract funnels. Let us check that it has the remaining two properties $\mathbf{S} 3$ and $\mathbf{S} 4$. Suppose $u \in V_{\zeta}\left[S\left(t_{0}, a\right)\right]$ and consider the shifted path $\sigma_{\tau} u$. By property $\mathbf{S} 3$ of the family $S, \sigma_{\tau} u \in S\left(t_{0}+\tau, u\left(t_{0}+\tau\right)\right)$. We have to show that $\sigma_{\tau} u$ maximizes $\zeta$ in the set $S\left(t_{0}+\tau, u\left(t_{0}+\tau\right)\right)$. Pick any path $v$ 
in $S\left(t_{0}+\tau, u\left(t_{0}+\tau\right)\right)$ and consider the spliced path $w=u \underset{t_{0}+\tau}{\bowtie} v$, which, by property $\mathbf{S} 4$, belongs to the funnel $S\left(t_{0}, a\right)$. Since $u$ maximizes $\zeta$ over $S\left(t_{0}, a\right), \zeta(u) \geq \zeta(w)$, i.e.,

$$
\int_{0}^{\infty} e^{-\lambda t} \varphi\left(u\left(t_{0}+t\right)\right) d t \geq \int_{0}^{\infty} e^{-\lambda t} \varphi\left(w\left(t_{0}+t\right)\right) d t
$$

But

$$
\begin{aligned}
& \int_{0}^{\infty} e^{-\lambda t} \varphi\left(w\left(t_{0}+t\right)\right) d t=\int_{0}^{\tau} e^{-\lambda t} \varphi\left(u\left(t_{0}+t\right)\right) d t+\int_{\tau}^{\infty} e^{-\lambda t} \varphi\left(v\left(t_{0}+t\right)\right) d t= \\
& \int_{0}^{\tau} e^{-\lambda t} \varphi\left(u\left(t_{0}+t\right)\right) d t+e^{-\lambda \tau} \int_{0}^{\infty} e^{-\lambda t} \varphi\left(v\left(t_{0}+\tau+t\right)\right) d t
\end{aligned}
$$

while

$$
\int_{0}^{\infty} e^{-\lambda t} \varphi\left(u\left(t_{0}+t\right)\right) d t=\int_{0}^{\tau} e^{-\lambda t} \varphi\left(u\left(t_{0}+t\right)\right) d t+e^{-\lambda \tau} \int_{0}^{\infty} e^{-\lambda t} \varphi\left(u\left(t_{0}+\tau+t\right)\right) d t .
$$

Hence,

$$
\int_{0}^{\infty} e^{-\lambda t} \varphi\left(u\left(t_{0}+\tau+t\right)\right) d t \geq \int_{0}^{\infty} e^{-\lambda t} \varphi\left(v\left(t_{0}+\tau+t\right)\right) d t
$$

which means $\sigma_{\tau} u$ is a maximizer in $S\left(t_{0}+\tau, u\left(t_{0}+\tau\right)\right)$. To check property $\mathbf{S} 4$ for $V_{\zeta}[S]$, pick $u \in V_{\zeta}\left[S\left(t_{0}, a\right)\right]$ and $v \in V_{\zeta}\left[S\left(t_{0}+\tau, u\left(t_{0}+\tau\right)\right)\right]$ and consider the spliced path $w=u \underset{t_{0}+\tau}{\bowtie} v$. We have to show that $w$ maximizes $\zeta$ over $S\left(t_{0}, a\right)$ and hence belongs to $V_{\zeta}\left[S\left(t_{0}, a\right)\right]$. This follows from a simple calculation that takes into account what we have just shown, that $\zeta\left(\sigma_{\tau} u\right)=m_{\zeta}\left(t_{0}+\tau, u\left(t_{0}+\tau\right)\right)$. If $v \in V_{\zeta}\left[S\left(t_{0}+\tau, u\left(t_{0}+\tau\right)\right)\right]$, then $\zeta(v)=m_{\zeta}\left(t_{0}+\tau, u\left(t_{0}+\tau\right)\right)$ as well. Thus,

$$
\begin{aligned}
& \int_{0}^{\infty} e^{-\lambda t} \varphi\left(w\left(t_{0}+t\right)\right) d t=\int_{0}^{\tau} e^{-\lambda t} \varphi\left(u\left(t_{0}+t\right)\right) d t+\int_{\tau}^{\infty} e^{-\lambda t} \varphi\left(v\left(t_{0}+t\right)\right) d t= \\
& \int_{0}^{\tau} e^{-\lambda t} \varphi\left(u\left(t_{0}+t\right)\right) d t+e^{-\lambda \tau} \zeta(v)=\int_{0}^{\tau} e^{-\lambda t} \varphi\left(u\left(t_{0}+t\right)\right) d t+e^{-\lambda \tau} \zeta\left(\sigma_{\tau} u\right)=\zeta(u),
\end{aligned}
$$

i.e., $\zeta(w)=m_{\zeta}\left(t_{0}, a\right)$, i.e., $w \in V_{\zeta}\left[S\left(t_{0}, a\right)\right]$. To summarize, for any functional $\zeta$ of the form (10), $V_{\zeta}\left[S\left(t_{0}, a\right)\right]$ is a family of abstract integral funnels.

Now, choose a countable family $\Phi$ of continuous functions $\varphi: X \rightarrow[0,1]$ that separates the points of $X$, see [4]. Choose some enumeration $\left(\lambda_{n}, \varphi_{n}\right)$ of the countable set of pairs $(\lambda, \varphi)$, where $\lambda$ runs through positive rational numbers and $\varphi$ runs through $\Phi$. To each pair $\left(\lambda_{n}, \varphi_{n}\right)$ corresponds the functional $\zeta_{n}$ via (10). Define recursively the shrinking families of abstract integral funnels

$$
S^{0}\left(t_{0}, a\right)=S\left(t_{0}, a\right), \quad S^{n}\left(t_{0}, a\right)=V_{\zeta_{n}}\left[S^{n-1}\left(t_{0}, a\right)\right], n=1,2, \ldots
$$

For each $\left(t_{0}, a\right), S^{n}\left(t_{0}, a\right)$ is a sequence of nested compacta in $\Omega^{t_{0}}$. The intersection,

$$
S^{\infty}\left(t_{0}, a\right)=\bigcap_{n=0}^{\infty} S^{n}\left(t_{0}, a\right)
$$


is not empty and compact. In fact, $S^{\infty}\left(t_{0}, a\right)$ is an abstract family of integral funnels. Indeed, it is easy to see that properties $\mathbf{S 1}, \mathbf{S 3}$, and $\mathbf{S 4}$, are satisfied. As the intersection of compact-valued maps into a Polish space, $S^{\infty}\left(t_{0}, \cdot\right)$ is measurable by [1, Lemma 18.4].

It turns out that each funnel $S^{\infty}\left(t_{0}, a\right)$ is a singleton. Indeed, if $u, v \in S^{\infty}\left(t_{0}, a\right)$, then, for every $\varphi \in \Phi$,

$$
\int_{0}^{\infty} e^{-\lambda t} \varphi\left(u\left(t_{0}+t\right)\right) d t=\int_{0}^{\infty} e^{-\lambda t} \varphi\left(v\left(t_{0}+t\right)\right) d t, \quad \forall \lambda \in \mathbb{Q}_{+} .
$$

By the uniqueness of the Laplace transform, $\varphi\left(u\left(t_{0}+t\right)\right)=\varphi\left(v\left(t_{0}+t\right)\right)$ for all $t \geq 0$. Because this is true for every $\varphi \in \Phi$ and the family $\Phi$ separates the points of $X$, we obtain $u\left(t_{0}+t\right)=v\left(t_{0}+t\right)$ for all $t \geq 0$, i.e., $u=v$ as paths.

For $u \in S^{\infty}\left(t_{0}, a\right)$, we will use the notation $u\left(t ; t_{0}, a\right)$, where $t \geq t_{0}$. If $t_{1}>t_{0}$, then $\left(\sigma_{t_{1}-t_{0}} u\right)(t)=u\left(t ; t_{1}, u\left(t_{1} ; t_{0}, a\right)\right)$ for $t \geq t_{1}$, by property S3. Thus, if $t_{2}>t_{1}$, $\left(\sigma_{t_{1}-t_{0}} u\right)\left(t_{2}\right)=u\left(t_{2} ; t_{1}, u\left(t_{1} ; t_{0}, a\right)\right)$. On the other hand, $\left(\sigma_{t_{1}-t_{0}} u\right)\left(t_{2}\right)=u\left(t_{2} ; t_{0}, a\right)$ by the definition of the shift operator $\sigma_{\tau}$. This establishes the semigroup property of the measurable selection $u\left(t ; t_{0}, a\right)$.

Once the (measurable) selection $u$ is found, we define the process $U\left(t_{1}, t_{0}\right): X \rightarrow X$ for $t_{1} \geq t_{0} \geq 0$, by the formula $U\left(t_{1}, t_{0}\right)(a)=u\left(t_{1} ; t_{0}, a\right)$. As we have shown, the map $a \mapsto\left\{u\left(\cdot ; t_{0}, a\right)\right\}$ from $X$ to $2^{\Omega}$ is measurable and singleton-valued. By the Kuratowski-RyllNardzewski selection theorem the map $a \mapsto u\left(\cdot ; t_{0}, a\right)$ from $X$ to $\Omega^{t_{0}}$ is $\left(\mathcal{B}_{X}, \mathcal{B}_{\Omega}\right)$-measurable. The map $U\left(t_{1}, t_{0}\right): X \rightarrow X$ is the composition of the map $a \mapsto u\left(\cdot ; t_{0}, a\right)$ and the evaluation map $\pi_{t_{1}}: \Omega^{t_{0}} \ni w \rightarrow \pi_{t_{1}}(w)=w\left(t_{1}\right) \in X$, which is continuous. Consequently, $U\left(t_{1}, t_{0}\right)$ is Borel measurable. This completes the proof.

\section{Local processes}

Let $X$ be a separable complete metric space as in the previous section. The integral funnels $S\left(t_{0}, a\right)$ will now be local. This means that to every initial state $a \in X$ and every initial moment $t_{0}$ corresponds a strictly positive number $T\left(t_{0}, a\right)$, the terminal time. The paths in the funnel $S\left(t_{0}, a\right)$ form a subset in $C\left(\left[t_{0}, t_{0}+T\left(t_{0}, a\right)\right) \rightarrow X\right)$.

Definition 2. A family $S\left(t_{0}, a\right), t_{0} \in[0,+\infty), a \in X$, will be called a family of abstract local integral funnels with terminal times $T\left(t_{0}, a\right)$ if they satisfy the following conditions.

TT $T\left(t_{0}, a\right)$ is a lower semi-continuous function on $[0,+\infty) \times X$, i.e., if $\left(t_{n}, a_{n}\right) \rightarrow\left(t_{0}, a\right)$, then $T\left(t_{0}, a\right) \leq \liminf T\left(t_{n}, a_{n}\right)$.

LS1 Every set $S\left(t_{0}, a\right)$ is a non-empty compact in the space $C\left(\left[t_{0}, t_{0}+T\left(t_{0}, a\right)\right) \rightarrow X\right)$ with the topology of uniform convergence on every closed subinterval $[\alpha, \beta]$ of $\left[t_{0}, t_{0}+\right.$ $\left.T\left(t_{0}, a\right)\right)$. Every path $w\left(\cdot ; t_{0}, a\right) \in S\left(t_{0}, a\right)$ is a continuous map from $\left[t_{0}, t_{0}+T\left(t_{0}, a\right)\right)$ into $X$, and $w\left(t_{0} ; t_{0}, a\right)=a$. 
LS2 For every $t_{0} \geq 0$, the set-valued map $a \mapsto S\left(t_{0}, a\right)$ is measurable in the following sense. Each path $w$ in $S\left(t_{0}, a\right)$ can be re-parametrized as $\tilde{w}(s)=w\left(t_{0}+s\left(T\left(t_{0}, a\right)-t_{0}\right)\right)$ and then viewed as an element of the space $\tilde{\Omega}=C([0,1) \rightarrow X)$. Denote by $\tilde{S}\left(t_{0}, a\right)$ the set $S\left(t_{0}, a\right)$ after such re-parametrization. We say that the map $a \mapsto S\left(t_{0}, a\right)$ is measurable if, for any closed subset $F$ of $\tilde{\Omega}$ (with the compact-open topology),

$$
\left\{a \in X: \tilde{S}\left(t_{0}, a\right) \cap F \neq \emptyset\right\} \in \mathcal{B}_{X}
$$

LS3 If $u \in S\left(t_{0}, a\right)$ and $\tau<T\left(t_{0}, a\right)$, then $T\left(t_{0}+\tau, u\left(t_{0}+\tau ; t_{0}, a\right)\right) \geq T\left(t_{0}, a\right)-\tau$ and $\sigma_{\tau} u \in S\left(t_{0}+\tau, u\left(t_{0}+\tau ; t_{0}, a\right)\right)$.

LS4 If $u \in S\left(t_{0}, a\right), \tau<T\left(t_{0}, a\right)$, and $v \in S\left(t_{0}+\tau, u\left(t_{0}+\tau ; t_{0}, a\right)\right)$, then the spliced path $w=u \underset{t_{0}+\tau}{\bowtie} v$, defined by analogy with (15), belongs to the funnel $S\left(t_{0}, a\right)$.

Theorem 2. Every family of local abstract integral funnels $S\left(t_{0}, a\right), t_{0} \in[0,+\infty), a \in X$, with terminal times $T\left(t_{0}, a\right)$, has a selection $\mathfrak{u}\left(\cdot ; t_{0}, a\right)$ with the following properties.

a) For every $t_{0} \geq 0$, the $\operatorname{map} X \ni a \mapsto \mathfrak{u}\left(\cdot ; t_{0}, a\right) \in C\left(\left[t_{0}, t_{0}+T\left(t_{0}, a\right)\right) \rightarrow X\right)$ is measurable.

b) $\mathfrak{u}\left(t_{0} ; t_{0}, a\right)=a$,

c) $\mathfrak{u}\left(t_{2} ; t_{1}, \mathfrak{u}\left(t_{1} ; t_{0}, a\right)\right)=\mathfrak{u}\left(t_{2} ; t_{0}, a\right)$ for all $t_{1} \in\left[t_{0}, t_{0}+T\left(t_{0}, a\right)\right)$ and $t_{2} \in\left[t_{1}, t_{0}+T\left(t_{0}, a\right)\right)$.

Proof. We mimic the proof of Theorem 1 with a few modifications. Let $\varphi: X \rightarrow[0,1]$ be a continuous function and let $\lambda$ be a positive real number. For $t_{0} \geq 0$ and $a \in X$, define the functional $\zeta$ on $S\left(t_{0}, a\right)$ via the formula

$$
\zeta(w)=\int_{0}^{T\left(t_{0}, a\right)} e^{-\lambda t} \varphi\left(w\left(t_{0}+t\right)\right) d t .
$$

This is a continuous functional and it attains its maximum on the compact set $S\left(t_{0}, a\right)$. Denote this maximum by $m_{\zeta}\left(t_{0}, a\right)$,

$$
m_{\zeta}\left(t_{0}, a\right)=\max _{w \in S\left(t_{0}, a\right)} \zeta(w)
$$

and define

$$
V_{\zeta}\left[S\left(t_{0}, a\right)\right]=\left\{v \in S\left(t_{0}, a\right): \zeta(v)=m_{\zeta}\left(t_{0}, a\right)\right\}
$$

The way we treat measurability by re-parametrizing the paths (see property LS2), allows us to apply the measurable maximum theorem, [1, Theorem 18.19], and conclude that $V_{\zeta}\left[S\left(t_{0}, a\right)\right]$ is a non-empty compact subset of $S\left(t_{0}, a\right)$, and the set-valued map $a \mapsto$ $V_{\zeta}\left[S\left(t_{0}, a\right)\right]$ is measurable. This shows that the family of sets $V_{\zeta}\left[S\left(t_{0}, a\right)\right]$ has properties LS1 and LS2. Suppose $u\left(\cdot ; t_{0}, a\right) \in V_{\zeta}\left[S\left(t_{0}, a\right)\right]$ and consider the shifted path $\sigma_{\tau} u$. By property LS3, $\sigma_{\tau} u \in S\left(t_{0}+\tau, u\left(t_{0}+\tau ; t_{0}, a\right)\right)$. Let us show that $\sigma_{\tau} u$ maximizes $\zeta$ in the set $S\left(t_{0}+\tau, u\left(t_{0}+\tau ; t_{0}, a\right)\right)$. Pick any path $v$ in $S\left(t_{0}+\tau, u\left(t_{0}+\tau ; t_{0}, a\right)\right)$ and consider the spliced 
path $w=u \underset{t_{0}+\tau}{\bowtie} v$, which, by property LS4, belongs to the funnel $S\left(t_{0}, a\right)$. Since $u$ maximizes $\zeta$ over $S\left(t_{0}, a\right), \zeta(u) \geq \zeta(w)$, i.e.,

$$
\int_{0}^{T\left(t_{0}, a\right)} e^{-\lambda t} \varphi\left(u\left(t_{0}+t ; t_{0}, a\right)\right) d t \geq \int_{0}^{T\left(t_{0}, a\right)} e^{-\lambda t} \varphi\left(w\left(t_{0}+t ; t_{0}, a\right)\right) d t .
$$

Now compute

$$
\begin{aligned}
& \int_{0}^{T\left(t_{0}, a\right)} e^{-\lambda t} \varphi\left(w\left(t_{0}+t ; t_{0}, a\right)\right) d t= \\
& \int_{0}^{\tau} e^{-\lambda t} \varphi\left(u\left(t_{0}+t ; t_{0}, a\right)\right) d t+\int_{\tau}^{T\left(t_{0}, a\right)} e^{-\lambda t} \varphi\left(v\left(t_{0}+t ; t_{0}+\tau, u\left(t_{0}+\tau ; t_{0}, a\right)\right) d t=\right. \\
& \int_{0}^{\tau} e^{-\lambda t} \varphi\left(u\left(t_{0}+t ; t_{0}, a\right)\right) d t+e^{-\lambda \tau} \int_{0}^{T\left(t_{0}, a\right)-\tau} e^{-\lambda t} \varphi\left(v\left(t_{0}+\tau+t ; t_{0}+\tau, u\left(t_{0}+\tau ; t_{0}, a\right)\right)\right) d t,
\end{aligned}
$$

and

$$
\begin{aligned}
& \int_{0}^{T\left(t_{0}, a\right)} e^{-\lambda t} \varphi\left(u\left(t_{0}+t ; t_{0}, a\right)\right) d t= \\
& \int_{0}^{\tau} e^{-\lambda t} \varphi\left(u\left(t_{0}+t ; t_{0}, a\right)\right) d t+e^{-\lambda \tau} \int_{0}^{T\left(t_{0}, a\right)-\tau} e^{-\lambda t} \varphi\left(u\left(t_{0}+\tau+t ; t_{0}, a\right)\right) d t .
\end{aligned}
$$

Hence,

$$
\begin{aligned}
& \zeta\left(\sigma_{\tau} u\right)=\int_{0}^{T\left(t_{0}, a\right)-\tau} e^{-\lambda t} \varphi\left(u\left(t_{0}+\tau+t ; t_{0}, a\right)\right) d t \geq \\
& \int_{0}^{T\left(t_{0}, a\right)-\tau} e^{-\lambda t} \varphi\left(v\left(t_{0}+\tau+t ; t_{0}+\tau, u\left(t_{0}+\tau ; t_{0}, a\right)\right)\right) d t=\zeta(v),
\end{aligned}
$$

which means $\sigma_{\tau} u$ is a maximizer in $S\left(t_{0}+\tau, u\left(t_{0}+\tau ; t_{0}, a\right)\right)$. With similar modifications, following the proof of Theorem 1, one verifies property $\mathbf{L S} 4$ for $V_{\zeta}[S]$. Thus, for any functional $\zeta$ of the form (13),$V_{\zeta}\left[S\left(t_{0}, a\right)\right]$ is a family of abstract local integral funnels.

Choose a countable family $\Phi$ of continuous functions $\varphi: X \rightarrow[0,1]$ that separates the points of $X$, and choose some enumeration $\left(\lambda_{n}, \varphi_{n}\right)$ of the countable set of pairs $(\lambda, \varphi)$, where $\lambda$ runs through positive rational numbers and $\varphi$ runs through $\Phi$. To each pair $\left(\lambda_{n}, \varphi_{n}\right)$ corresponds the functional $\zeta_{n}$ via (13). Define recursively the shrinking families of abstract integral funnels $S^{0}\left(t_{0}, a\right)=S\left(t_{0}, a\right)$, and $S^{n}\left(t_{0}, a\right)=V_{\zeta_{n}}\left[S^{n-1}\left(t_{0}, a\right)\right], n=1,2, \ldots$ Again, the intersection,

$$
S^{\infty}\left(t_{0}, a\right)=\bigcap_{n=0}^{\infty} S^{n}\left(t_{0}, a\right)
$$

is an abstract family of local integral funnels. To show that each funnel $S^{\infty}\left(t_{0}, a\right)$ is a singleton, assume $u, v \in S^{\infty}\left(t_{0}, a\right)$ Then, for every $\varphi \in \Phi$,

$$
\int_{0}^{T\left(t_{0}, a\right)} e^{-\lambda t} \varphi\left(u\left(t_{0}+t ; t_{0}, a\right)\right) d t=\int_{0}^{T\left(t_{0}, a\right)} e^{-\lambda t} \varphi\left(v\left(t_{0}+t ; t_{0}, a\right)\right) d t, \quad \forall \lambda \in \mathbb{Q}_{+} .
$$


By the uniqueness of the Laplace transform, $\varphi\left(u\left(t_{0}+t ; t_{0}, a\right)\right)=\varphi\left(v\left(t_{0}+t ; t_{0}, a\right)\right)$ for all $t \in\left[0, T\left(t_{0}, a\right)\right)$. Because this is true for every $\varphi \in \Phi$ and the family $\Phi$ separates the points of $X$, we obtain $u\left(t_{0}+t ; t_{0}, a\right)=v\left(t_{0}+t ; t_{0}, a\right)$ for all $t \in\left[0, T\left(t_{0}, a\right)\right)$, i.e., $u=v$ as paths.

For the unique path in the funnel $S^{\infty}\left(t_{0}, a\right)$, let us use the notation $\mathfrak{u}\left(t ; t_{0}, a\right)$. If $t_{0}<t_{1}<$ $T\left(t_{0}, a\right)$, then $\left(\sigma_{t_{1}-t_{0}} \mathfrak{u}\right)(t)=\mathfrak{u}\left(t ; t_{1}, \mathfrak{u}\left(t_{1} ; t_{0}, a\right)\right)$ for $t_{1} \leq t<T\left(t_{0}, a\right)$, by property LS3. Thus, if $t_{2}>t_{1},\left(\sigma_{t_{1}-t_{0}} \mathfrak{u}\right)\left(t_{2}\right)=\mathfrak{u}\left(t_{2} ; t_{1}, \mathfrak{u}\left(t_{1} ; t_{0}, a\right)\right)$. On the other hand, $\left(\sigma_{t_{1}-t_{0}} \mathfrak{u}\right)\left(t_{2}\right)=\mathfrak{u}\left(t_{2} ; t_{0}, a\right)$ by the definition of the shift operator $\sigma_{\tau}$. This establishes the semigroup property of the measurable selection $\mathfrak{u}\left(t ; t_{0}, a\right)$. The fact that the maps $X \ni a \mapsto \mathfrak{u}\left(\cdot ; t_{0}, a\right) \in C\left(\left[t_{0}, T\left(t_{0}, a\right)\right) \rightarrow X\right.$ are measurable is established by an argument with the distance function similar to the one in the end of the proof of Theorem 1. This completes the proof.

Remark 3. There are differential equations for which not all initial conditions $x\left(t_{0}\right)=x_{0}$ are possible: there is a proper subset $C \subset(-\infty,+\infty) \times X$ of allowed initial conditions. This is the case, i.e., for the Clairaut equations. We discuss a particular, illustrative example in the next section. However, it is not hard to see that Theorems 1 and 2 can be adapted for such situations.

Remark 4. We can introduce the local process maps $U\left(t_{1}, t_{0}\right)$ as in the previous section: $U\left(t_{1}, t_{0}\right)(a)=\mathfrak{u}\left(t_{1} ; t_{0}, a\right)$. However, now $U\left(t_{1}, t_{0}\right)$ may not be defined on all of $X$ and, for every $a$, the range of admissible $t_{1}$ will be different. If we restrict $t_{0}$ to a compact set $[\alpha, \beta] \subset[0,+\infty)$ and a to a compact set $K \subset X$, then, thanks to assumption $\mathbf{T T}$, the infimum of $T\left(t_{0}, a\right)$ over $[\alpha, \beta] \times K$ is strictly positive, and this gives a non-trivial admissible interval for $t_{1}$ when $\left(t_{0}, a\right) \in[\alpha, \beta] \times K$. At the moment we do not see any benefits in constructing an abstract theory of such local (semi)-processes.

\section{Example}

The Clairaut equation is a scalar ODE of the form

$$
x=t \dot{x}+\psi(\dot{x})
$$

with some function $\psi$. The standard method of solution is as follows. Differentiate (16),

$$
\dot{x}=\dot{x}+t \ddot{x}+\psi^{\prime}(\dot{x}) \ddot{x}
$$

and simplify the result to obtain

$$
\ddot{x}\left(t+\psi^{\prime}(\dot{x})\right)=0 \text {. }
$$

This offers two possibilities:

$$
\ddot{x}=0
$$

and/or

$$
t+\psi^{\prime}(\dot{x})=0 .
$$


Solve each of the equations with the initial condition $x\left(t_{0}\right)=x_{0}$. The first equation implies

$$
x(t)=x_{0}+c\left(t-t_{0}\right)
$$

with a constant $c$. Substitute this into the original equation (16):

$$
x_{0}+c\left(t-t_{0}\right)=t c+\psi(c) \text {. }
$$

This yields the equation for the possible value(s) of $c$ :

$$
x_{0}=c t_{0}+\psi(c) \text {. }
$$

Assuming $c\left(t_{0}, x_{0}\right)$ is a solution of (20) (there may be many solutions), we can re-write the solution (19) as follows:

$$
x(t)=\psi\left(c\left(t_{0}, x_{0}\right)\right)+c\left(t_{0}, x_{0}\right) t .
$$

The second equation (18) requires some kind of invertibility of the function $\psi^{\prime}$ (the simplest case is when $\psi^{\prime \prime} \neq 0$, i.e., $\psi$ is convex or concave). Assuming the inverse function $\left(\psi^{\prime}\right)^{-1}$ makes sense, we obtain

$$
\dot{x}=\left(\psi^{\prime}\right)^{-1}(-t)
$$

After integration,

$$
x(t)=x_{0}+\int_{t_{0}}^{t}\left(\psi^{\prime}\right)^{-1}(-s) d s .
$$

Substitute this expression into (16):

$$
x_{0}+\int_{t_{0}}^{t}\left(\psi^{\prime}\right)^{-1}(-s) d s=t\left(\psi^{\prime}\right)^{-1}(-t)+\psi\left(\left(\psi^{\prime}\right)^{-1}(-t)\right)
$$

Notice that

$$
\frac{d}{d s}\left[s\left(\psi^{\prime}\right)^{-1}(-s)+\psi\left(\left(\psi^{\prime}\right)^{-1}(-s)\right)\right]=\left(\psi^{\prime}\right)^{-1}(-s)
$$

Thus, equation (24) can be simplified to

$$
x_{0}-\left[t_{0}\left(\psi^{\prime}\right)^{-1}\left(-t_{0}\right)+\psi\left(\left(\psi^{\prime}\right)^{-1}\left(-t_{0}\right)\right)\right]=0
$$

It is useful to introduce the following version of the Legendre transform of the function $\psi$ :

$$
\tilde{\psi}\left(t_{*}\right)=\inf _{t}\left[t_{*} \cdot t+\psi(t)\right] .
$$

Then equation (25) can be written in the form

$$
x_{0}=\tilde{\psi}\left(t_{0}\right)
$$

and the solution (23) takes the form

$$
x(t)=x_{0}+\tilde{\psi}(t)-\tilde{\psi}\left(t_{0}\right) .
$$


In view of (27), we obtain

$$
x(t)=\tilde{\psi}(t) .
$$

This is the so-called singular solution. We see that the singular solution corresponds to the value of $c=c\left(t_{0}, x_{0}\right)$ in (21) that minimizes $[c \cdot t+\psi(c)]$.

Consider the special case $\psi(s)=s^{2}$, i.e., consider the equation

$$
x=t \dot{x}+(\dot{x})^{2} .
$$

The corresponding equation (20) has two solutions for $c$,

$$
c_{ \pm}\left(t_{0}, x_{0}\right)=-\frac{t_{0}}{2} \pm \sqrt{\frac{t_{0}^{2}}{4}+x_{0}},
$$

provided $x_{0}+t_{0}^{2} / 4>0$, has one solution when $x_{0}+t_{0}^{2} / 4=0$, and has no solutions when $x_{0}+t_{0}^{2} / 4<0$. Since $\tilde{\psi}\left(t_{*}\right)=-t_{*}^{2} / 4$, the singular solution is

$$
x(t)=-\frac{t^{2}}{4}
$$

Thus, the region $C \subset(-\infty,+\infty) \times \mathbb{R}$ of the allowed initial data $\left(t_{0}, x_{0}\right)$ is the parabola $x=-t^{2} / 4$ and the points above it:

$$
C=\left\{(t, x): x \geq-\frac{t^{2}}{4}\right\}
$$

The parabola is the envelope of the straight lines (19) or, equivalently, (21):

$$
x(t)=c^{2}+c t .
$$

Through every point $\left(t_{0}, x_{0}\right)$ in the interior of $C$ pass two straight lines (solutions) corresponding to $c_{+}\left(t_{0}, x_{0}\right)$ and $c_{-}\left(t_{0}, x_{0}\right)$. It is useful to notice that one of the lines (32) touches the parabola in the past (at some moment $t=\mathfrak{t}_{p}\left(t_{0}, x_{0}\right)<t_{0}$ ) and the other touches the parabola in the future (at some moment $\left.t=\mathfrak{t}_{f}\left(t_{0}, x_{0}\right)>t_{0}\right)$. Let us denote these lines (solutions) $\mathfrak{x}_{p}\left(t ; t_{0}, x_{0}\right)$ and $\mathfrak{x}_{f}\left(t ; t_{0}, x_{0}\right)$, respectively. They are defined for all $t \in \mathbb{R}$. The integral funnel $S\left(t_{0}, x_{0}\right)$ contains the rays $\mathfrak{x}_{p}\left(t ; t_{0}, x_{0}\right)$ and $\mathfrak{x}_{f}\left(t ; t_{0}, x_{0}\right)$ for $t \geq t_{0}$. In addition, it contains infinitely many solutions that branch off $\mathfrak{x}_{f}\left(t ; t_{0}, x_{0}\right)$ : once the line touches the parabola at $t=\mathfrak{t}_{f}\left(t_{0}, x_{0}\right)$, the trajectory may continue along the parabola forever, or, at any time $r>\mathfrak{t}_{f}\left(t_{0}, x_{0}\right)$ it may take off along the tangent line.

To every point $\left(t_{0}, x_{0}\right)$ on the boundary of $C$ (on the parabola) correspond infinitely many solutions of (29) that can be divided into three categories: 1) there is the singular solution (31); 2) there is a ray tangent to the parabola:

$$
\mathfrak{x}_{p f}\left(t ; t_{0},-\frac{t_{0}^{2}}{4}\right)=-\frac{t_{0}}{2} t+\frac{t_{0}^{2}}{4}, \quad t \geq t_{0} ;
$$


3) there is a one-parameter family of solutions that follow the parabola for some time and then get off on the tangent line:

$$
x_{r}(t)= \begin{cases}-\frac{t^{2}}{4} & \text { for } \quad t_{0} \leq t \leq r \\ -\frac{r}{2} t+\frac{r^{2}}{4} & \text { for } t \geq r .\end{cases}
$$

All these solutions form the funnel $S\left(t_{0}, x_{0}\right)$ when $\left(t_{0}, x_{0}\right)$ is on the parabola.

It is not hard to see that the trajectories that stay on the parabola for some time and then leave it cannot be part of the semi-process. Thus, there are three choices of a semiprocess corresponding to equation (29). The first choice is completely determined by the initial conditions on the parabola: for $\left(t_{*}, x_{*}\right) \in \partial C$,

$$
\mathfrak{u}\left(t ; t_{*}, x_{*}\right)=-\frac{t_{*}}{2} t+\frac{t_{*}^{2}}{4} \quad \forall t \geq t_{*} .
$$

When the point $\left(t_{*}, x_{*}\right)$ slides along the parabola clockwise, the corresponding rays $\mathfrak{x}_{p f}\left(t ; t_{*}, x_{*}\right)=$ $\mathfrak{u}\left(t ; t_{*}, x_{*}\right)$ sweep the interior of $C$. For every point $\left(t_{0}, x_{0}\right)$ in the interior of $C$, there is a unique point $\left(t_{*}, x_{*}\right)$ (with $t_{*}=\mathfrak{t}_{p}\left(t_{0}, x_{0}\right)$ ) on the parabola such that $\mathfrak{u}\left(t_{0} ; t_{*}, x_{*}\right)=x_{0}$. As a consequence, $\mathfrak{u}\left(t ; t_{0}, x_{0}\right)=\mathfrak{u}\left(t ; t_{*}, x_{*}\right)$ describes the ray-solution starting at $\left(t_{0}, x_{0}\right)$.

The second choice of the semi-process coincides with the first one on the interior of $C$, while on the parabola we pick the singular solution: $\mathfrak{u}\left(t ; t_{0},-t_{0}^{2} / 4\right)=-t^{2} / 4$.

The third choice is to select the trajectories (rays) that touch the parabola in the future and then continue along the parabola. In other words,

$$
\mathfrak{u}\left(t ; t_{0}, x_{0}\right)=-\frac{t^{2}}{4} \quad \text { if } \quad x_{0}=-\frac{t_{0}^{2}}{4}
$$

and, if $\left(t_{0}, x_{0}\right)$ is in the interior of $C$, then

$$
\mathfrak{u}\left(t ; t_{0}, x_{0}\right)= \begin{cases}\mathfrak{x}_{f}\left(t ; t_{0}, x_{0}\right) & t_{0} \leq t \leq \mathfrak{t}_{f}\left(t_{0}, x_{0}\right), \\ -\frac{t^{2}}{4} & t>\mathfrak{t}_{f}\left(t_{0}, x_{0}\right)\end{cases}
$$

All three semi-processes are Borel measurable. Depending on the choice of a countable family

$\Phi$ of continuous bounded functions that separate the points of $X=\mathbb{R}$, the maximization procedure described in the proof of Theorem 1 will pick out one of the three semi-processes.

\section{References}

[1] Ch. Aliprantis and K. Border. Infinite Dimensional Analysis. A Hitchhiker's Guide. 3rd Edition. Springer-Verlag, Berlin Heidelberg, 2006.

[2] Claude Berge, Topological Spaces: Including a Treatment of Multi-Valued Functions, Vector Spaces and Convexity. Dover Books on Mathematics. Reprint of the Oliver \& Boyd, Edinburgh and London, 1963 edition. 
[3] N. P. Bhatia and O. Hájek. Local Semi-Dynamical Systems. Lecture Notes in Mathematics, vol. 90. Springer, 1969

[4] D. Blount and M. A. Kouritzin. On convergence determining and separating classes of functions. Stochastic Processes and Their Applications, 120 (2010) 1898-1907

[5] Jorge E. Cardona. On Statistical Solutions of Nonlinear Evolution Equations. Ph.D. Thesis, University of Miami, Coral Gables, June 2017.

[6] Jorge E. Cardona and L. Kapitanski. Semiflow selection and Markov selection theorems. arXiv:1707.04778

[7] Dafermos, Constantine M. An invariance principle for compact processes. J. Differential Equations 9 (1971), 239-252; erratum, ibid. 10 (1971), 179-180

[8] F. Flandoli, M. Romito, Markov selections for the 3D stochastic Navier-Stokes equations, Probab. Theory Related Fields 140 (2008) 407-458

[9] B. Goldys, M. Röckner, X. Zhang, Martingale solutions and Markov selections for stochastic partial differential equations, Stochastic Processes and their Applications Volume 119, Issue 5, May 2009, Pages 1725-1764

[10] Hájek, Otomar. Theory of processes. I. Czechoslovak Math. J. 17 (92) 1967 159-199

[11] Hájek, Otomar. Theory of processes. II. Czechoslovak Math. J. 17 (92) 1967 372-398.

[12] Jack Hale. Theory of Functional Differential Equations. Springer-Verlag, New York - Heidelberg - Berlin, 1977

[13] S. Hu and N. S. Papageorgiou. Handbook of Multivalued Analysis. Volume I: Theory. Mathematics and Its Applications Vol. 149, Kluwer Academic Publishers, 1997.

[14] A. D. Ioffe. Survey of measurable selection theorems: Russian literature supplement. SIAM J. Control Opt. 16 (1978) no. 5, 728-732.

[15] L. Kapitanski and S. Živanović Gonzalez, Attractors in hyperspace, Topological Methods in Nonlinear Analysis Volume 44, No. 1, 2014, 199-227

[16] H. Kneser, Über die Lösungen eines Systems gewöhnlicher Differentialgleichungen, das der Lipschitzschen Bedingung nicht genügt, S. B. Preuss. Akad, 4 (1923), 171174.

[17] N. V. Krylov, On the selection of a Markov process from a system of processes and the construction of quasi-diffusion processes, Izv. Akad. Nauk SSSR Ser. Mat. 37 (1973) No. 3, 691-708

[18] K. Kuratowski. Topology, vol.1. Academic Press, 2014. 
[19] K. Kuratowski and C. Ryll-Nardzewski, A general theorem on selectors, Bull. Acad. Pol. Sc. 13 (1965) 397-403

[20] T. Parthasarathy. Selection Theorems and Their Applications. Lecture Notes in Mathematics (Book 263), Springer, 1972.

[21] Robert J. Sacker and George R. Sell. Skew-product flows, finite extensions of minimal transformation groups and almost periodic differential equations. Bulletin of the AMS, vol. 79, number 4, July 1973, 802-805

[22] G. R. Sell, Nonautonomous differential equations and topological dynamics. I, II, Trans. Amer. Math. Soc. 127 (1967), 241-262, 263-283

[23] G. R. Sell, Differential equations without uniqueness and classical topological dynamics, Journal of Differential Equations 14, (1973) 42-56

[24] D.W. Stroock, S.R.S. Varadhan, Multidimensional Diffusion Processes, SpringerVerlag, Berlin, 1979.

[25] Daniel H. Wagner. Survey of measurable selection theorems. SIAM J. Control Optimization, vol. 15, no. 5 (1977) 859-903. 